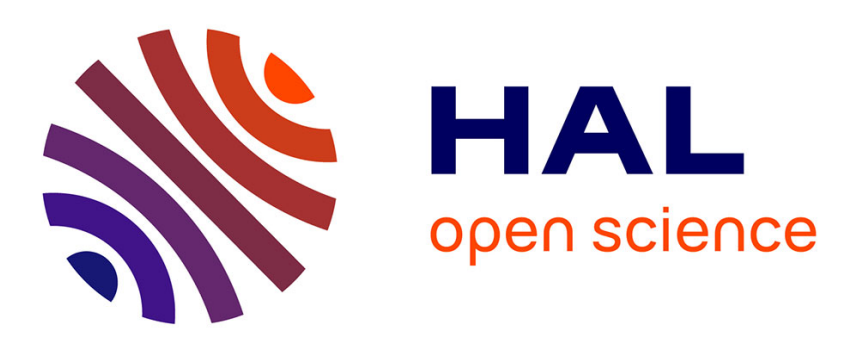

\title{
A two level optimization approach for long-term planning in a large air transportation network
} Amadou Handou, Aïcha Alou Oumarou, Catherine Mancel, Felix Mora-Camino

\section{- To cite this version:}

Amadou Handou, Aïcha Alou Oumarou, Catherine Mancel, Felix Mora-Camino. A two level optimization approach for long-term planning in a large air transportation network. IPDO 2007, 2nd Symposium on Inverse Problems, Design and Optimization, Apr 2007, Miami Beach, United States. pp 275-286, 10.1080/17415970802166733 . hal-00938152

\section{HAL Id: hal-00938152 \\ https://hal-enac.archives-ouvertes.fr/hal-00938152}

Submitted on 22 May 2014

HAL is a multi-disciplinary open access archive for the deposit and dissemination of scientific research documents, whether they are published or not. The documents may come from teaching and research institutions in France or abroad, or from public or private research centers.
L'archive ouverte pluridisciplinaire HAL, est destinée au dépôt et à la diffusion de documents scientifiques de niveau recherche, publiés ou non, émanant des établissements d'enseignement et de recherche français ou étrangers, des laboratoires publics ou privés. 


\section{A TWO LEVEL OPTIMIZATION APPROACH FOR LONG TERM PLANNING IN A LARGE AIR TRANSPORTATION NETWORK}

\author{
Amadou Handou \\ World Food Program, \\ Via Cesare Giulio Viola 68/70 \\ Parco de Medici,00148 Roma, Italy \\ Amadou.handou@wfp.org \\ Catherine Mancel \\ LARA/ENAC, Air Transportation Department \\ 7,Avenue Edouard Belin, \\ 31055 Toulouse, France \\ catherine.mancel@enac.fr
}

\author{
Aïcha Alou Oumarou \\ LARA/ENAC, Air Transportation Departmen,t \\ 7,Avenue Edouard Belin, \\ 31055 Toulouse, France \\ aicha.alou-oumarou@enac.frr \\ Félix Mora-Camino \\ LARA/ENAC, Air Transportation Department, \\ 7 Avenue Edouard Belin, \\ 31055,,Toulouse, France \\ felix.mora@enac.fr
}

\section{ABSTRACT}

In this communication is considered the problem of long term forecasting of the traffic growth in a large air transportation network. This problem is crucial when planning the necessary investments in airports, fleets and air traffic control equipments.

The proposed approach makes use of two different optimization models: One model is devoted to demand forecasting, the other one defines the air transport supply according with a profit maximization behavior for the airlines sector.

A proposed solution scheme is composed of an iterative process between the current solutions of the demand and the supply optimization problems. Convergence conditions are discussed for this iterative process between these two problems which can be seen as inverse of each other.

The proposed optimization approach is briefly illustrated in the case of the long term forecasting of air traffic in the west African region.

\section{INTRODUCTION}

In this communication is considered the problem of long term forecasting of the traffic growth in a large air transportation network. This problem is crucial when planning the necessary investments in airports, fleets and air traffic control resources. One of the main difficulty of this task is related with the estimation of future demand over the network which has direct influence on the operational conditions and costs.

The proposed approach makes use of two different optimization models: One model is devoted to demand forecasting, the other one defines the air transport supply according with a profit maximization behavior for the whole airlines sector operating in this area.

The demand forecasting process is composed of a mix of possibilistic (fuzzy) and probabilistic considerations since fuzzy modeling is adopted to characterize scenarios while a new entropy maximization approach is proposed to determine scenario based origin-destination matrices.

The supply optimization model considers simultaneously two classes of flows: aircraft flows providing air transportation capacity and passengers flows generating revenues to the airlines operators. Hence, no classical flows in networks optimization technique is available to solve this problem while two level solution techniques considering aircraft flows at the first level and passengers flows at the other can be considered. Here also the large degree of uncertainty with respect to operations costs is taken into account through possibilistic scenarios Then a global optimization problem is associated to each full scenario with respect to demand and operations costs. 
Each of the two optimization problems, taken separately is convex, however, the whole problem, through the definition of the revenue and of a global cost constraint is non convex.

A proposed solution schemes is composed of an iterative process between the current solutions of the demand and the supply optimization problems: the entropy maximizing problem provides the passengers origin-destination matrix given a fare structure, while the supply optimization problem provides the fare structure given a passengers origindestination matrix. To enforce convergence while maintaining convexity of the two problems, the effective-potential demand level constraints of the supply optimization problem are modified.

The proposed optimization approach for the long term forecasting problem is illustrated considering the case of West Africa.

\section{DISTRIBUTION OF DEMAND}

To perform the prevision of demand, it is supposed that a priori trip distribution is described by fuzzy matrices $\left[\widetilde{T}_{i j}\right]$ where the $\widetilde{T}_{i j}$ are fuzzy numbers. This information may be the result of a fuzzy prediction of trip distribution for a previous time period or the result of local forecasting studies. It is also supposed [1] that fuzzy predictions of generation and attraction potential levels $\widetilde{O}_{i}, i \in\{1, \ldots, N\}$ and $\widetilde{D}_{j}, j \in\{1, \ldots, N\}$, are available.

\section{Local cost elasticities}

We consider that the demand for a given origin destination $i-j$ is such that there exists a positive constant $\lambda_{i j}$ with :

$$
T_{i j}\left(\bar{\pi}_{i j}\right)=T_{i j}\left(\bar{\pi}_{i j}^{0}\right) e^{-\lambda_{i j}\left(\bar{\pi}_{i j}-\bar{\pi}_{i j}^{0}\right)}
$$

where $\bar{\pi}_{i j}$ is a mean fare between $i$ and $j$. Considering that the potential origin and destination levels $O_{i}, i \in\{1, \ldots, N\}$ and $D_{j}, j \in\{1, \ldots, N\}$ are such as :

$$
\begin{gathered}
\sum_{j=1, j \neq i}^{N} T_{i j}(0)=O_{i} \quad i=1 \grave{a} N \\
\sum_{i=1, i \neq j}^{N} T_{i j}(0)=D_{j} \quad j=1 \grave{a} N
\end{gathered}
$$

we have the following constraints:

$$
\sum_{j=1, j \neq i}^{N} T_{i j} e^{\lambda \bar{\pi}_{i j}}=O_{i} \quad i=1 \grave{a} N
$$

$$
\sum_{i=1, i \neq j}^{N} T_{i j} e^{\lambda \bar{\pi}_{i j}}=D_{j} \quad j=1 \grave{a} N
$$

Here matrix $\left[\lambda_{i j}\right]$ is supposed to be described in fuzzy terms by a fuzzy membership function $\mu_{\lambda}\left(\left[\lambda_{i j}\right]\right) \cdot$ Observe that a more general relation for (1) could be:

$$
T_{i j}\left(\bar{\pi}_{i j}\right) f_{i j}\left(\bar{\pi}_{i j}\right)=T_{i j}\left(\bar{\pi}_{i j}^{0}\right) f_{i j}\left(\bar{\pi}_{i j}^{0}\right)
$$

where $f_{i j}$ is a continuous positive monotonous decreasing function such as:

$$
f_{i j}(0)=1 \text { and } \lim _{x \rightarrow+\infty} f_{i j}(x)=0
$$

\section{Estimation of demand distribution}

The demand distribution estimation problem is taken here as a constrained entropy maximization problem. Then to a choice of an instance $I=\left\{\left[\hat{T}_{i j}\right]\right.$, $\left.O_{i}, i \in\{1, \ldots, N\}, \quad D_{j}, j \in\{1, \ldots, N\},\left[\lambda_{i j}\right]\right\}$ is associated the following maximization problem, Problem I:

$$
\max \quad-\sum_{i=1}^{N} \sum_{j=1, j \neq i}^{N} T_{i j} \operatorname{Ln}\left(T_{i j} / \hat{T}_{i j}\right)
$$

under constraints (4), (5) and

$$
T_{i j} \geq 0, i=1 \grave{a} N, j=1 \grave{a} N, i \neq j
$$

The adopted optimization criteria, a conditional entropy function, is representative of the global distortion between the a priori and the predicted demand distributions. To its solution $\left[T_{i j}{ }^{*}\right]$ is attached a global possibilistic degree $\mu_{I}$ given by:

$$
\mu_{I}=\left(\prod_{i=1}^{N} \mu_{O_{i}}\left(O_{i}\right)\right)\left(\prod_{j=1}^{N} \mu_{D_{j}}\left(D_{j}\right)\right)\left(\prod_{i=1}^{N} \prod_{j=1, j \neq i}^{N} \mu_{T_{i j}}\left(\hat{T}_{i j}\right)\right) \mu_{\lambda}
$$

The instance is said to be consistent if the following condition is fulfilled :

$$
\sum_{i=1}^{N} O_{i}=\sum_{j=1}^{N} D_{j}=T
$$

and it is said highly believable if it is consistent and $\mu_{I}=1$. Of course highly believable solutions will be of primary interest to define central predictions, but to evaluate the degree of uncertainty of the future demand distribution, less believable solutions must be also obtained.

\section{Analysis of the solution}

The above optimization problem being convex it is useful to introduce the Lagrangian associated to this problem : 


$$
\begin{aligned}
& L=-\sum_{i=1}^{N} \sum_{j=1, j \neq i}^{N} T_{i j} \operatorname{Ln}\left(T_{i j} / \hat{T}_{i j}\right) \\
& +\sum_{i=1}^{N} \alpha_{i}\left(\sum_{j=1, j \neq i}^{N} T_{i j} e^{\lambda \bar{\pi}_{i j}}-O_{i}\right) \\
& +\sum_{j=1}^{N} \beta_{j}\left(\sum_{i=1, i \neq j}^{N} T_{i j} e^{\lambda \bar{\pi}_{i j}}-D_{j}\right)
\end{aligned}
$$

The first order optimality conditions are such as :

$$
\frac{\partial L}{\partial T_{i j}}=0 \quad i=1 \grave{a} N, j=1 \grave{a} N, i \neq j
$$

or :

$$
\begin{gathered}
-1-\operatorname{Ln}\left(T_{i j} / \hat{T}_{i j}\right)+\alpha_{i} e^{\lambda_{i j} \bar{\pi}_{i j}}+\beta_{j} e^{\lambda \bar{\pi}_{i j}}=0 \\
i=1 \grave{a} N, j=1 \grave{a} N, i \neq j
\end{gathered}
$$

Then the solution is such as :

$$
\begin{gathered}
T_{i j}^{*}=\hat{T}_{i j} e^{-\left(1+\left(\alpha_{i}+\beta_{j}\right) e^{\lambda_{i j} \bar{\pi}_{i j}}\right)} \\
i=1 \grave{a} N, j=1 \grave{a} N, i \neq j
\end{gathered}
$$

In the case that $\lambda_{i j} \bar{\pi}_{i j}$ is very small, this expression can be approximated by:

$$
\begin{aligned}
& T_{i j}^{*}=\hat{T}_{i j} e^{-\left(1+\left(\alpha_{i}+\beta_{j}\right)\left(1+\lambda_{i j} \bar{\pi}_{i j}\right)\right)} \\
& i=1 \grave{a} N, j=1 \grave{a} N, i \neq j
\end{aligned}
$$

The dual variables are such as:

$$
\begin{gathered}
\sum_{j=1, j \neq i}^{N} \theta_{i j} p_{i j}\left(\alpha_{i}+\beta_{j}\right)=O_{i} \quad i=1 \text { to } N \\
\sum_{i=1, i \neq j}^{N} \theta_{i j} p_{i j}\left(\alpha_{i}+\beta_{j}\right)=D_{j} \quad j=1 \text { to } N
\end{gathered}
$$

where

$$
\theta_{i j}=\hat{T}_{i j} e^{\left(\lambda_{i j} \bar{\pi}_{i j}-1\right)} \text { and } p_{i j}=e^{e^{\lambda_{i j} \bar{\pi}_{i j}}}
$$

however, the numerical solution of an instance of Problem I will be obtained using linear -convex algorithm such as the Simplex-Convex or FrankWolfe algorithm [2]. Another interesting direction for numerical resolution is through the solution of the geometric primal associated to this problem [3].

\section{AIRLINES SUPPLY MODEL}

The proposed model takes into account two types of flows and their corresponding constraints [4], [5]: aircraft flows along the airlines network according to the available fleets and passengers flows using the resulting transport capacity [7]. The fleet of aircraft and its operation generate fixed and variable costs, while passengers flows are the main source of revenue for the airlines sector.

\section{Airline network model}

Considering that the airlines fleet is composed of medium and long range aircraft, to each operated airport $\mathrm{i}, \mathrm{i} \in \boldsymbol{A}$, is associated two sets of airports : $\boldsymbol{A}_{\boldsymbol{C}}(\mathrm{i})$ composed of the medium range airports reachable from airport i and $\boldsymbol{A}_{\boldsymbol{L}}(\mathrm{i})$ composed of the long range airports reachable from airport i. Then the medium and long range networks can be introduced:

$$
\boldsymbol{R}_{\boldsymbol{C}}=\left[\boldsymbol{G}_{\boldsymbol{C}},\left[f_{i j}^{c}\right]\right]
$$

where $\boldsymbol{G}_{\boldsymbol{C}}$ is the graph $\left[\boldsymbol{A}, \Gamma_{\boldsymbol{C}}\right]$ where the successor function $\Gamma_{C}$ is such as :

$$
\Gamma_{\boldsymbol{C}}(i)=\boldsymbol{A}_{\boldsymbol{C}}(i) \quad i \in \boldsymbol{A}
$$

[ $\left.f_{i j}^{c}\right]$ is the flow of medium range aircraft over $\boldsymbol{G}_{\boldsymbol{C}}$. It satisfies to conservation and positive ness constraints:

$$
\begin{gathered}
\sum_{j=1, j \neq i}^{N} f_{j i}^{c}=\sum_{k=1, k \neq i}^{N} f_{i k}^{c} \\
f_{i j}^{c} \geq 0, \quad i, j \in \boldsymbol{A}
\end{gathered}
$$

Here flows integrity constraints are not taken into account since no scheduling or routing problem will be formulated according to these flows which should only provide a global view of the future development of the air transportation network. However for sake of realism, a medium range fleet capacity constraint is introduced:

$$
\sum_{i=1}^{N} \sum_{j \in A_{c}(i)} f_{i j}^{c} d_{i j}^{c} \leq D_{c} F_{c}
$$

where $d_{i j}^{c}$ is the block time for a medium range aircraft for a flight between airports $i$ and $j, D_{c}$ is the medium time availability of a medium range aircraft. In the same way the long range network is such as :

$$
\boldsymbol{R}_{L}=\left[\boldsymbol{G}_{L},\left[f_{i j}^{L}\right]\right]
$$

with :

$$
\boldsymbol{G}_{\boldsymbol{L}}=\left[\boldsymbol{A}, \Gamma_{\boldsymbol{L}}\right], \Gamma_{\boldsymbol{L}}(i)=\boldsymbol{A}_{\boldsymbol{L}}(i) i \in \boldsymbol{A}
$$

$\left[f_{i j}^{L}\right]$ is the flow of long range aircraft over $\boldsymbol{G}_{\boldsymbol{L}}$, it is such as: 


$$
\begin{gathered}
\sum_{j=1, j \neq i}^{N} f_{j i}^{L}=\sum_{k=1, k \neq i}^{N} f_{i k}^{L} \\
f_{i j}^{L} \geq 0, \quad i, j \in \boldsymbol{A} \\
\sum_{i=1}^{N} \sum_{j \in A_{L}(i)} f_{i j}^{L} d_{i j}^{L} \leq D_{L} F_{L}
\end{gathered}
$$

where $d_{i j}^{L}$ is the block time for a long range aircraft for a flight between airports $i$ and $j, D_{L}$ is the medium time availability of a medium range aircraft. It can be also of interest to introduce ATC and airport capacity constraints such as respectively:

$\sum_{i=1}^{N} \sum_{j \in A_{L}(i)} S_{L}(i, j, t) f_{i j}^{L}+\sum_{i=1}^{N} \sum_{j \in A_{c}(i)} S_{c}(i, j, t) f_{i j}^{c} \leq Q_{T}^{t} \quad t \in T$

where $Q_{T}^{t}$ is the traffic capacity of ATC control sector $t, T$ being the set of traffic control sectors covering the whole network. $S_{L}$ and $S_{c}$ are incidence matrices between flights and control sectors:

$S_{L}(i, j, t)=1$ if long range flights between $i$ and $j$ cross ATC control sector $t$, else $S_{L}(i, j, t)=0$.

$S_{c}(i, j, t)=1$ if medium range flights between $i$ and $j$ cross traffic control sector $t$, else $S_{c}(i, j, t)=0$.

and for airport capacities:

$$
\begin{aligned}
& \sum_{j \in \Gamma_{c}^{-1}(i)} \omega_{a c} f_{j i}^{c}+\sum_{j \in \Gamma_{L}^{-1}(i)} \omega_{a L} f_{j i}^{L} \leq K_{i}^{a} \quad i \in A \\
& \sum_{j \in \Gamma_{c}(i)} \omega_{d c} f_{i j}^{c}+\sum_{j \in \Gamma_{L}(i)} \omega_{d L} f_{i j}^{L} \leq K_{i}^{d} \quad i \in A
\end{aligned}
$$

where $K_{i}^{a}$ is the arrival capacity at airport $i$ and $K_{i}^{d}$ is the departure capacity at airport $j$. Here $\omega_{a c}, \omega_{a L}$, $\omega_{d c}, \omega_{d L}$ are capacity parameters attached to the different types of operations (arrival or departure) and fleets (medium and long range fleets).

\section{Passengers flows modeling}

The medium range and long range aircraft networks provide the physical support for the passengers network defined as :

$$
\boldsymbol{R}_{p a x}=\left[\boldsymbol{G}_{p a x},\left[\phi_{i j}\right]\right]
$$

where $\boldsymbol{G}_{\boldsymbol{p a x}}=\left[\boldsymbol{A}, \boldsymbol{\Gamma}_{\boldsymbol{p a x}}\right]$ and $\boldsymbol{\Gamma}_{\boldsymbol{p a x}}$ is such as:

$$
\Gamma_{p a x}(i)=\boldsymbol{A}_{\boldsymbol{C}}(i) \cup \boldsymbol{A}_{L}(\mathrm{i}) i \in \boldsymbol{A}
$$

$\left[\phi_{i j}\right]$ is the passengers flows over this network. It obeys to the following capacity constraints :

$$
0 \leq \phi_{i j} \leq C_{i j} \quad i, j \in \boldsymbol{A}
$$

with

$C_{i j}=C_{c} f_{i j}^{C}+C_{L} f_{i j}^{L} \quad$ if $(i, j)$ is an $\operatorname{arc}$ of $\boldsymbol{G}_{\boldsymbol{C}}$ and $\boldsymbol{G}_{\boldsymbol{L}}$ $C_{i j}=C_{c} f_{i j}^{C} \quad$ if $(i, j)$ is an exclusive $\operatorname{arc}$ of $\boldsymbol{G}_{\boldsymbol{C}}$ and $C_{i j}=C_{L} f_{i j}^{L}$ if $(i, j)$ is an exclusive arc of $\boldsymbol{G}_{\boldsymbol{L}}$.

Within graph $\boldsymbol{G}_{\boldsymbol{p a x}}$ for each origin-destination pair a set of concurrent paths composed of a succession of flights is retained according to directness criteria:

$$
C h_{i j}=\left\{C h^{n}{ }_{i j}, \mathrm{n}=1 \text { to } \mathrm{n}_{\max }\right\} \quad \mathrm{i}, \mathrm{j} \in \boldsymbol{A}
$$

Here it is considered that airlines assign demand for a given O-D pair between these different possible paths according with their available seat capacity. This approach is acceptable when available capacity is close to demand levels.

Let $[\alpha(i, j, n, k, l)]$ be the incidence matrix between path $C h^{n}{ }_{i j}$ and arc $(\mathrm{k}, 1)$ of $\boldsymbol{G}_{\boldsymbol{p a x}}: \quad \alpha(i, j, n, k, l)=1$ if $(k, l) \in \mathrm{Ch}_{i j}^{n} \alpha(i, j, n, k, l)=0$ else. Then the flow of passengers between airports $i$ and $j$ is given by :

$\phi_{i j}=\sum_{k=1}^{N} \sum_{l=1, l \neq k}^{N}\left(\sum_{n=1}^{N_{i j}} \alpha(k, l, n, i, j) \theta_{k \ln }\right) \quad i, j \in A, i \neq j$

where $\theta_{i j n}$ is the flow of passengers between airports $i$ and $j$ using the $\mathrm{n}^{\text {th }}$ path between them.

The mean fare for the $i-j$ origin-destination pair is then given by :

$$
\bar{\pi}_{i j}=\left(\sum_{n=1}^{N_{i j}} \pi_{i j}^{n} \theta_{i j n}\right) /\left(\sum_{n=1}^{N_{i j}} \theta_{i j n}\right) \quad i, j \in A
$$

\section{SUPPLY OPTIMIZATION MODEL}

Given a distribution of potential demand $\left[T_{i j}^{*}\right]$, associated to an instance of $\left[\hat{T}_{i j}\right] \quad O_{i}, i \in\{1, \ldots, N\}$, $D_{j}, j \in\{1, \ldots, N\}$, and $\left[\lambda_{i j}\right]$, as well as to mean air transportation fares $\left[\bar{\pi}_{i j}\right]$, the optimization of the supply (capacities and fares) by the airlines can be 
considered: Problem II is concerned with the optimization of the global economic performance over a future period of time of the airlines operating the air transportation network.

Here the decision variables are the aircraft flows ( $f_{i j}^{C}$ and $f_{i j}^{L}$ ) between the different airports and the fares $\left(\pi_{i j}^{n}\right)$ applied to each selected path between the airports. To solve this problem it is also necessary to introduce the effective flows of passengers $\left(\theta_{i j n}\right)$ associated to each selected path between the airports.

The optimization criterion of Problem II is given by:

$$
\begin{gathered}
\max _{f_{i j}, \tau_{i j}^{n}, \theta_{i j}}\left(\sum_{i=1}^{N} \sum_{j \in \Gamma_{p a n}} \sum_{i=1}^{N_{i j}} \pi_{i j}^{n} \theta_{i j n}\right) /\left(1+\tau_{r}\right)- \\
\left(\left(c_{c f}+c_{c v} F_{c}+\sum_{i=1}^{N} \sum_{j \in_{c}} c_{i j}^{c v} f_{i j}^{c}\right)+\left(c_{L f}+c_{L v} F_{L}+\sum_{i=1}^{N} \sum_{j \in \Gamma_{c}}^{L v} c_{i j}^{L v} f_{i j}^{L}\right)\right)
\end{gathered}
$$

where $\tau$ is a return rate and the $c_{u v}$ are coefficients related with fixed and variable fleet and aircraft flows costs. The cost incertitude should be defined in a fuzzy framework an contribute to global scenarios involving demand and supply.

Problem II must satisfy the following constraints:

$$
\begin{gathered}
\sum_{j=1, i \in A_{c}(j)}^{N} f_{j i}^{c}=\sum_{k=1, k \in A_{c}(i)}^{N} f_{i k}^{c} \quad i, j \in \boldsymbol{A} \\
\sum_{j=1, i \in A_{L}(j)}^{N} f_{j i}^{L}=\sum_{k=1, k \in A_{L}(i)}^{N} f_{i k}^{L} \quad i, j \in \boldsymbol{A} \\
\sum_{i=1}^{N} \sum_{j \in A_{c}(i)} f_{i j}^{c} \Theta_{i j}^{c} \leq T_{c} F_{c} \\
\sum_{i=1}^{N} \sum_{j \in A_{L}(i)} f_{i j}^{L} \Theta_{i j}^{L} \leq T_{L} F_{L}
\end{gathered}
$$$$
\sum_{k=1}^{N} \sum_{l=1, l \neq k}^{N} \sum_{n=1}^{N_{H}} \alpha(k, l, n, i, j) \theta_{k \ln } \leq C_{c} f_{i j}^{C}+C_{L} f_{i j}^{L}
$$$$
\text { if }(i, j) \text { is an } \operatorname{arc} \text { of } \boldsymbol{G}_{\boldsymbol{C}} \text { and of } \boldsymbol{G}_{\boldsymbol{L}}
$$

$$
\sum_{k=1}^{N} \sum_{l=1, l \neq k}^{N} \sum_{n=1}^{N_{k l}} \alpha(k, l, n, i, j) \theta_{k \ln } \leq C_{c} f_{i j}^{C}
$$

if $(i, j)$ is exclusively an $\operatorname{arc}$ of $\boldsymbol{G}_{\boldsymbol{C}}$

$$
\sum_{k=1}^{N} \sum_{l=1, l \neq k}^{N} \sum_{n=1}^{N_{k}} \alpha(k, l, n, i, j) \theta_{k \ln } \leq C_{L} f_{i j}^{L}
$$

if $(i, j)$ is exclusively an $\operatorname{arc}$ of $\boldsymbol{G}_{\boldsymbol{L}}$

$$
\sum_{n=1}^{N_{i j}} \theta_{i j n} \leq T_{i j}^{*} \quad i, j \in \boldsymbol{A}, i \neq j
$$

with the positiveness conditions:

$$
\begin{gathered}
\theta_{k \ln } \geq 0 \quad k, l \in A, n \in N_{k l} \\
f_{i j}^{C} \geq 0, f_{i j}^{L} \geq 0 \quad i, j \in A \\
\pi_{i j}^{n} \geq 0 \quad i, j \in A, n \in N_{i j}
\end{gathered}
$$

\section{GLOBAL SOLUTION SCHEME}

It appears that Problem I and Problem II are strongly interdependent: while Problem I provides to Problem II potential levels of demand $\left[T_{i j}^{*}\right]$ (constraint (47)), Problem II provides mean fare values $\left[\bar{\pi}_{i j}\right]$ (relation (38)) to Problem I.

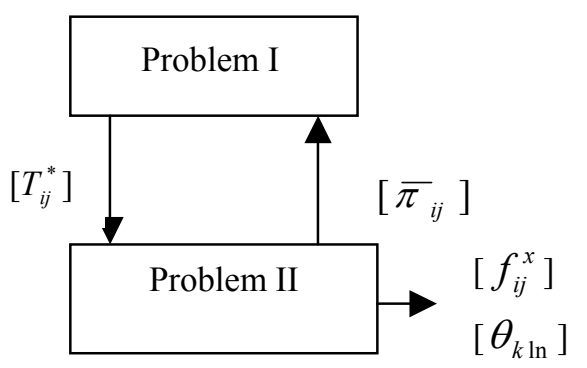

Figure 1: Interaction between problems

Each of the two optimization problems, taken separately is a convex programming problem, however, the whole problem when integrating Problem I into Problem II through constraint (47) is non convex. Then it seems interesting to solve numerically these problems separately and design an interactive process towards equilibrium. In this case, some questions are of interest:

- the easiness to solve numerically each of the optimization problems,

- the guarantee of convergence of the iterative process,

- the speed of convergence,

- the quality of the limit solution. 
These two linked problems constitute a non standard bilevel programming problem [7] where Problem II is the leader's problem while Problem $I$ is the follower's problem. This results from the adoption of a deregulated point of view in which airlines operate an air transport network according to their direct economic interest without considering any social surplus.

Constraint (47) plays a central role in the articulation of the two problems and the convergence of their solutions towards a common global solution. Since this constraints transmit to the revenue optimization problem the reaction of demand with respect to changes in mean fares between the different origins and destinations, it is useful to makes apparent this effect so that limited fare values will be provided by the solutions of Problem II. However, the relation between origin-destinations flows $\left[T_{i j}^{*}\right]$ and fare levels $\left[\bar{\pi}_{i j}\right]$ is quite complex and at least non linear. So, to maintain the convexity of Problem II, this constraint is replaced by its first order approximation where the reference values are the solutions of the two problems at the previous iteration:

$$
\begin{gathered}
\sum_{n=1}^{N_{i j}} \theta_{i j n} \leq T_{i j}^{*(k-1)} \\
\left.+\sum_{l=1}^{N} \sum_{m=1, m \neq l}^{N} \frac{\partial T_{i j}^{*}}{\partial \bar{\pi}_{l m \mid k-1}^{*}} \sum_{n=1}^{N_{l m}} \frac{\partial \bar{\pi}_{l m}}{\partial \pi_{l m \mid k-1}^{n}}\left(\pi_{l m}^{n}-\bar{\pi}_{l m}^{n}(k-1)\right)\right) \\
i, j \in A, \quad i \neq j
\end{gathered}
$$

The partial derivatives present in relation (51) can be computed by differentiating relations (15), (17), (18) and (38) at the solutions of Problem I and II at iteration $k-1$ and solving linear systems of equations. The following inequalities are guaranteed:

$$
\left.\begin{array}{l}
\frac{\partial T_{i j}^{*}}{\partial \bar{\pi}_{i j} \mid k-1} \leq 0, \frac{\partial T_{i j}^{*}}{\partial \bar{\pi}_{l m} \mid k-1} \geq 0 \\
\left|\frac{\partial T_{i j}^{*}}{\left.\partial \bar{\pi}_{l m}\right|_{k-1}}\right| \ll\left|\frac{\partial T_{i j}^{*}}{\partial \bar{\pi}_{i j}}\right| k-1 \mid
\end{array}\right\}
$$

$i, j \in A, \quad i \neq j, l, m \in A, \quad l \neq m,(l, m) \neq(i, j)$

So it appears that starting from low fares, an increase of fares on trips linking origin $i$ and destination $j$ at solution of Problem II at iteration $k-1$ will imply a decrease of potential demand on the same origindestination pair at the same iteration of Problem I and then a negative effect on the increase of airlines revenue. Fares will then be increased by this process until no more improvement of revenue is obtained. Starting from high level fares, the inverse process will be obtained.

This iterative process can be displayed in a very simple example of optimization problem:

$$
\left.\begin{array}{c}
\max _{\pi, \theta} \pi \theta-c \theta \\
\text { under } \quad \theta \leq \theta_{0} e^{-\lambda \pi}
\end{array}\right\}
$$

where $\theta_{0}$ and $\lambda$ are positive constants. Its exact solution is given by: $\pi^{*}=(1+\mathrm{c} \lambda) / \lambda$ and $\theta^{*}=\theta_{0} e^{-(1+\lambda c)}$. Similarly to (51), its feasible set can be approximated around $\left(\pi_{\mathrm{k}-1}, \theta_{k-1}\right)$ by:

$$
\theta \leq \theta_{k-1}\left(1+\lambda \pi_{k-1}\right)-\lambda \theta_{k-1} \pi
$$

and the solution of problem $\left\{\max _{\pi, \theta} \pi \theta-c \theta\right.$ under $(54)\}$ is :

$$
\left\{\begin{array}{c}
\pi_{k}=\left(1+\lambda \pi_{k-1}+\lambda c\right) /(2 \lambda) \\
\theta_{k}=\theta_{0} \exp \left(-\lambda \pi_{k}\right)
\end{array}\right.
$$

It is clear that the limit when $\mathrm{k} \rightarrow+\infty$ of $\left(\pi_{k}, \theta_{k}\right)$ is $\left((1+\mathrm{c} \lambda) / \lambda, \theta_{0} \mathrm{e}^{-(1+\mathrm{c} \lambda}\right)$. Here, the convergence is monotonous and its rate is such as:

$$
\left(\pi_{k}-\pi_{k-1}\right)=(1 / 2)\left(\pi_{k-1}-\pi_{k-2}\right)
$$

Other bilevel schemes have been considered in [8] and [9] for different transportation problems while numerical convergence conditions have been discussed in [10].

\section{APPLICATION TO A REGIONAL CASE}

The proposed modeling approach has been applied to the case of West Africa composed of 16 countries.

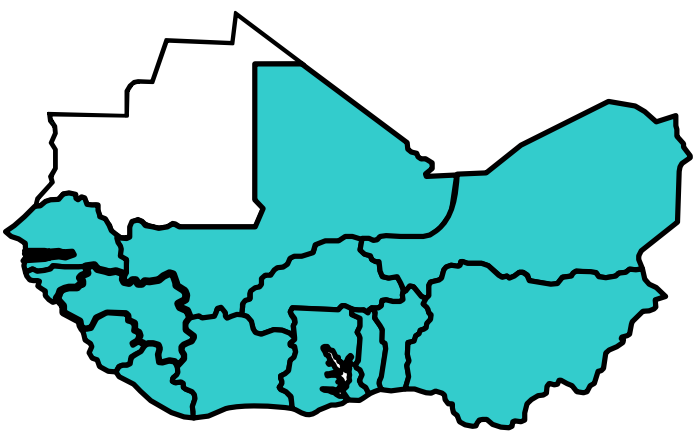

Figure 2: Ouest Africa region 
The considered network [11] is composed of 7 main local (Lagos, Abidjan, Accra, Dakar, Ouagadougou, Bamako, Niamey) and 5 main external nodes ( Paris, Londres, New York, Djeddah, Johannesburg) for a total of 40 links. This leads to consider in the formulation of Problem II: 50 aircraft flows, 187 passengers flows and fares, 132 potential demand levels, 18 fleet conservation flows constraints, 2 fleet capacity constraints, 45 passengers flows capacity constraints, 187 demand levels constraints and 556 positiveness constraints. This is a medium size optimization problem by actual standards. However the solution of this problem will be recalled repetitively in two loops: one related with the solution of the global problem for an instance of the input data, the other will be related with the exploration of the space of possible scenarios and corresponding fuzzy solutions.

\section{CONCLUSION}

This communication has considered the problem of long term forecasting of the traffic growth in a large air transportation network.

The proposed approach has introduced two different optimization models: One model devoted to demand forecasting and the other describing a profit maximization supply behavior by airlines.

The demand forecasting process is composed of a mix of possibilistic and probabilistic considerations since fuzzy modeling is adopted to characterize scenarios while an entropy maximization approach is used to determine origin-destination matrices. The formulation of this problem introduces in a new way elasticity of demand with respect to fares. The supply optimization model considers simultaneously two classes of flows: aircraft flows providing air transportation capacity and passengers flows generating revenues to the airlines operators. Here also the large degree of uncertainty with respect to operations costs is taken into account through possibilistic scenarios. Then a global optimization problem is associated to each full scenario with respect to demand and operations costs.

The proposed solution scheme is composed of an iterative process between the current solutions of the demand and the supply optimization problems: the entropy maximizing problem provides the passengers origin-destination matrix given a fare structure, while the supply optimization problem provides the fare structure given a passengers origindestination matrix. Convergence conditions are discussed for this iterative process between two problems which can be seen as inverse of each other. The proposed modeling approach has been illustrated considering the case of West Africa over the next decades, but full numerical results need to be obtained so that the whole approach can be fully evaluated.

\section{REFERENCES}

1. Charfeddine S., Mora-Camino F. et de Coligny M., "Fuzzy linear regression: Application to the estimation of air transport demand". Proc. Int. Conf. on Fuzzy Sets and Soft Computing in Economics and Finance, Saint Petersburg. ISBN 968-489-030-3, pp. 350-359, 2004

2. Assad, A.A.," Multicommodity network flowsa survey", Networks, 8, pp. 37-91, 1978.

3. Mora-Camino F., «Introduction à la Programmation Géométrique », Editora COPPE, Rio de Janeiro, 1978.

4. Berge C., "The theory of graphs", London, Dover, 2001.

5. Ford L.R. and D.R. Fulkerson, "Flows in networks", Princeton, Princeton University Press, 1962.

6. Alou A. R. Kaffa and F. Mora-Camino, «A multilevel modelling approach for air transportation system »,Laboratoire d'Automatique et de Recherche Opérationnelle-LARA,ENAC ,July 2006.

7. Dempe S., "Foundations of bilevel programming". Kluwer Academic Publishers, Dordrecht, 2000.

8. Alou A. , R. Kaffa and F. Mora-Camino, «Pricing in air transport systems: a multilevel approach », XIV Congreso Panamericano de Trafico y Transporte, Las Palmas de Gran Canaria, September 2006.

9. Brotcorne L., M. Labbé, P. Marcotte and G. Savard, "A bilevel model for toll optimization on a multicommodity transportation network", Transportation Science, vol. 35, pp.1-14, (2000).

10. Scheel H. and S. Scholtes, “ Mathematical programs with equilibrium constraints: stationarity, optimality and sensitivity". Mathematics of Operations Research vol.25, pp.1-22, 2000.

11. Handou A., "Contribution à l'Optimisation d'un Réseau de Transport Aérien:Proposition d'un Modèle basé sur la Logique Floue et la Maximisation Entropique », PhD dissertation, LARA/ENAC, Toulouse, December 2006. 